\title{
Remarks on Wilson Loops and Seifert Loops in Chern-Simons Theory
}

\author{
Chris Beasley
}

\begin{abstract}
As noted long ago by Atiyah and Bott, the classical Yang-Mills action on a Riemann surface admits a beautiful symplectic interpretation as the norm-square of a moment map associated to the Hamiltonian action by gauge transformations on the affine space of connections. Here I will explain how certain Wilson loop observables in Chern-Simons gauge theory on a Seifert three-manifold can be given an analogous symplectic description. Among other results, this symplectic description implies that the stationary-phase approximation to the Wilson loop path integral is exact for torus knots, an empirical observation made previously by Lawrence and Rozansky. This article reviews selected material from the larger work "Localization for Wilson Loops in Chern-Simons Theory," arXiv:0911.2687.
\end{abstract}

\section{Introduction}

This brief article is intended as an introduction to the study of Chern-Simons gauge theory via non-abelian localization [4, 5. I will not attempt to give here a comprehensive overview of the subject. Instead, my goal is to highlight two very beautiful ideas, one old and one new, which enter the story of non-abelian localization in an essential way and which may have applications elsewhere. See also [6, 15] for alternative approaches to path integral localization in Chern-Simons theory.

To set the stage, we consider Chern-Simons theory on a compact, oriented threemanifold $M$ with gauge group $G$. By assumption $G$ will be a compact, connected, simply-connected, and simple Lie group. For instance, $G$ could be $S U(N)$ for any $N>1$. These assumptions on $G$ ensure that any principal $G$-bundle $P$ over $M$ is topologically trivial, a technical convenience. The gauge field $A$ of Chern-Simons theory is then a connection on $P$.

Let me now introduce the Wilson loop operators in Chern-Simons theory. Quite generally, a Wilson loop operator $W_{R}(C)$ in any gauge theory on a manifold $M$ is described by the data of an oriented, closed curve $C$ which is smoothly $\sqrt{1}$ embedded

2000 Mathematics Subject Classification. Primary 81T45, Secondary 53D20 57M27.

${ }^{1}$ The condition that $C$ be smoothly embedded in $M$ is merely for convenience and is not strictly required to define $W_{R}(C)$ as a sensible operator in gauge theory. Indeed, the Wilson loop expectation value in Chern-Simons theory can be computed exactly even for the case that $C$ is an arbitrary closed graph 25 in $M$. 
in $M$ and which is decorated by an irreducible representation $R$ of the gauge group $G$. As a classical functional of the connection $A$, the Wilson loop operator $W_{R}(C)$ is then given simply by the trace in $R$ of the holonomy of $A$ around $C$,

$$
\begin{aligned}
W_{R}(C) & =\operatorname{Tr}_{R} \operatorname{Hol}_{C}(A), \\
& =\operatorname{Tr}_{R} P \exp \left(-\oint_{C} A\right) .
\end{aligned}
$$

Following the standard practice in physics, we describe the holonomy $\operatorname{Hol}_{C}(A)$ in the second line of (1.1) in terms of a path-ordered exponential $P \exp \left(-\oint_{C} A\right)$, which describes solutions to the first-order differential equation for parallel transport2 along $C$.

With the Wilson loop operator in hand, we finally introduce the absolutelynormalized Wilson loop path integral

$$
Z(k ; C, R)=\frac{1}{\operatorname{Vol}(\mathcal{G})} \int_{\mathcal{A}} \mathcal{D} A W_{R}(C) \exp \left[i \frac{k}{4 \pi} \operatorname{CS}(A)\right] .
$$

Here $\operatorname{CS}(A)$ is the Chern-Simons action,

$$
\mathrm{CS}(A)=\int_{M} \operatorname{Tr}\left(A \wedge d A+\frac{2}{3} A \wedge A \wedge A\right),
$$

and ' $\mathrm{Tr}$ ' denotes a suitably-normalized, negative-definite, invariant quadratic form on the Lie algebra $\mathfrak{g}$ of $G$. For instance, if $G$ is $S U(N)$, the quadratic form ' $T r$ ' is given concretely by the trace in the fundamental $N$-dimensional representation of $S U(N)$.

For later use, let me recall three essential properties of the Chern-Simons action. First, the Chern-Simons action is purely topological, insofar as it depends only on the choice of an orientation, not a metric, on $M$. Second, the critical points of the Chern-Simons action are precisely the flat connections on $M$, for which

$$
F_{A}=d A+A \wedge A=0 .
$$

Finally, even though the Chern-Simons action is not manifestly gauge-invariant and indeed the Chern-Simons action is not gauge-invariant - the Chern-Simons action is almost gauge-invariant, in the sense that it is invariant under homotopically trivial gauge transformations. Under homotopically nontrivial gauge transformations, the functional $\operatorname{CS}(A)$ shifts by integral multiples of $8 \pi^{2}$, where the relevant integer is determined by the homotopy class of the gauge transformation as a map from $M$ to $G$. So long as the Chern-Simons level $k \in \mathbb{Z}$ is quantized to be an integer, the exponential of the Chern-Simons action in (1.2) is then honestly gauge-invariant.

Otherwise, we present $Z(k ; C, R)$ as an integral over the infinite-dimensional affine space $\mathcal{A}$ of connections on $M$. As usual in gauge theory, we divide that integral by the volume of the infinite-dimensional group $\mathcal{G}$ of gauge transformations acting on $\mathcal{A}$.

Before proceeding, let me make one elementary remark. When $R$ is the trivial representation, the Wilson loop operator $W_{R}(C)$ is the identity operator, and the

\footnotetext{
${ }^{2}$ Because we work in conventions for which $d_{A}=d+A$ is the covariant derivative, a minus sign appears in the argument of the path-ordered exponential.
} 
absolutely-normalized Wilson loop path integral reduces immediately to the path integral which describes the Chern-Simons partition function $Z(k)$ on $M$,

$$
Z(k)=\frac{1}{\operatorname{Vol}(\mathcal{G})} \int_{\mathcal{A}} \mathcal{D} A \exp \left[i \frac{k}{4 \pi} \operatorname{CS}(A)\right]
$$

In terms of $Z(k)$ and $Z(k ; C, R)$, the Wilson loop expectation value $\left\langle W_{R}(C)\right\rangle$ is then given by the ratio

$$
\left\langle W_{R}(C)\right\rangle=\frac{Z(k ; C, R)}{Z(k)} .
$$

Though the Wilson loop expectation value is very convenient to consider for certain purposes, we work exclusively with $Z(k ; C, R)$ today.

As it stands, the Wilson loop path integral in (1.2) is a purely formal expression. Nonetheless, over twenty years ago Witten 24 gave a completely precise prescription to define the observable $Z(k ; C, R)$, based upon the canonical quantization of Chern-Simons theory in the Hamiltonian formalism. This formalism leads to a well-known algebraic description $2 \mathbf{2 3}$ of $Z(k ; C, R)$ in terms of a presentation of $M$ via surgery on $S^{3}$, combined with data about certain modular representations associated to two-dimensional rational conformal field theories.

Beyond providing a rigorous means to define the observable $Z(k ; C, R)$, the Hamiltonian formalism is also very powerful, insofar as it can be used to perform explicit, exact calculations in Chern-Simons theory. For instance, among the classic results in 24, Witten computed the unknot observable $Z(k ; \bigcirc, \boldsymbol{j})$ for ChernSimons theory on $S^{3}$ with gauge group $S U(2)$,

$$
Z(k ; \bigcirc, j)=\sqrt{\frac{2}{k+2}} \sin \left(\frac{\pi j}{k+2}\right), \quad j=1, \ldots, k+1 .
$$

Here $\boldsymbol{j}$ is the irreducible representation of $S U(2)$ with dimension $j$, and as indicated, $j$ runs without loss over the finite set of representations which are integrable in the affine Lie algebra for $S U(2)$ at level $k$.

Yet despite its computability, the algebraic definition of $Z(k ; C, R)$ in the Hamiltonian formalism obscures many features which are manifest in the preceding path integral (1.2) and which one would like to understand more deeply. As a simple example, in the semi-classical limit that $k$ is large, a naive stationary-phase approximation can be applied to the path integral describing the Chern-Simons partition function, and this approximation implies asymptotic behavior for $Z(k)$ that is far from evident in the complicated, exact expressions that arise from conformal field theory. Nonetheless, the predicted asymptotic behavior can be checked in examples, as was done early on by Freed and Gompf [10, Jeffrey [13, and Garoufalidis [1]. See for instance $\S 7$ of $[20$ for a survey of continuing work in this area.

This article concerns a very special and very beautiful situation in which the stationary-phase approximation to the Wilson loop path integral is actually exact. Namely, the three-manifold $M$ is a Seifert manifold, equipped with a distinguished locally-free $U(1)$ action, and the curve $C$ is a Seifert fiber of $M$. By definition, a locally-free $U(1)$ action is one for which the generating vector field is nowhere vanishing. Equivalently, all stabilizers under a locally-free $U(1)$ action are proper, necessarily discrete, subgroups of $U(1)$.

Let me introduce a bit of terminology. The existence of a locally-free $U(1)$ action implies that the Seifert manifold $M$ decomposes geometrically as the total 
space of a nontrivial circle bundle over a Riemann surface $\Sigma$,

$$
\begin{aligned}
S^{1} \longrightarrow & M \\
& \downarrow \\
& \downarrow
\end{aligned}
$$

Here $\Sigma$ is allowed to have orbifold points, and the circle bundle is allowed to be a corresponding orbifold bundle, so long as $M$ itself is smooth.

Each Seifert manifold carries a distinguished set of Wilson loop operators which respect the $U(1)$ action by rotations in the fiber of (1.8). For these Wilson loops, the curve $C$ is an orbit of the given $U(1)$ action, and $C$ appears geometrically in $M$ as the $S^{1}$ fiber over a basepoint $\sigma \in \Sigma$. Assuming that $\sigma$ is a smooth (non-orbifold) point of $\Sigma$, the topology of $C$ in $M$ does not depend upon the choice of $\sigma$, so such Wilson loops are determined entirely by the choice of the representation $R$. Henceforth, we refer to these special Wilson loop operators which wrap the generic Seifert fibers of $M$ as "Seifert loop operators" to distinguish them from general Wilson loops in $M$, about which we will also have some things to say.

As a concrete example, $S^{3}$ admits countably-many locally-free $U(1)$ actions, each associated to a distinct Seifert presentation. According to a basic result of Moser [19], the corresponding knots which can be realized as Seifert fibers in $S^{3}$ are precisely the torus knots. So the Seifert loop operators in $S^{3}$ are just the Wilson loop operators which wrap torus knots.

\section{Some Experimental Evidence}

The exactness of the stationary-phase approximation to the Seifert loop path integral was discovered by Lawrence and Rozansky [16 on an empirical basis, through a detailed analysis of the explicitly-known formulae for $Z(k ; C, R)$ in the case $G=S U(2) 3$ Specifically, after a rather involved series of algebraic manipulations, Lawrence and Rozansky were able to rewrite $Z(k ; C, R)$ very compactly as a finite sum of analytic expressions, each being either a contour integral or the residue of a meromorphic function. These summands in $Z(k ; C, R)$ could then be associated in a one-to-one fashion with the connected components in the moduli space of flat connections on $M$. Since the flat connections on $M$ are the critical points of the Chern-Simons action, such a form for $Z(k ; C, R)$ strongly suggests that the stationary-phase approximation to the Seifert loop path integral is exact.

By way of illustration, Lawrence and Rozansky would rewrite the exact formula for the unknot observable $Z(k ; \bigcirc, j)$ in (1.7) as

$$
\begin{aligned}
& Z(k ; \bigcirc, j)= \\
& \quad \frac{1}{2 \pi i} \mathrm{e}^{-\frac{i \pi\left(1+j^{2}\right)}{2(k+2)}} \int_{-\infty}^{+\infty} d x \operatorname{ch}_{j}\left(\mathrm{e}^{\frac{i \pi}{4}} \frac{x}{2}\right) \sinh ^{2}\left(\mathrm{e}^{\frac{i \pi}{4}} \frac{x}{2}\right) \exp \left(-\frac{(k+2)}{8 \pi} x^{2}\right) .
\end{aligned}
$$

Here $\mathrm{ch}_{\boldsymbol{j}}$ is the character of $S U(2)$ associated to the representation $\boldsymbol{j}$,

$$
\operatorname{ch}_{j}(y)=\frac{\sinh (j y)}{\sinh (y)}=\mathrm{e}^{(j-1) y}+\mathrm{e}^{(j-3) y}+\cdots+\mathrm{e}^{-(j-3) y}+\mathrm{e}^{-(j-1) y},
$$

and the equality between the expressions in (1.7) and (1.9) follows by evaluating (1.9) as a sum of elementary Gaussian integrals.

\footnotetext{
${ }^{3}$ See 12, 17 for the generalization to other gauge groups $G$.
} 
Of course, the only flat connection on $S^{3}$ is the trivial connection. As a result, the aforementioned sum in $Z(k ; \bigcirc, \boldsymbol{j})$ contains only a single term, given by the integral over the real variable $x$ in (1.9). According to Lawrence and Rozansky, this integral is to be interpreted as the stationary-phase contribution from the trivial connection to the full Wilson loop path integral in (1.2).

One of the main results in 4, 5 is to make the semi-classical interpretation of formulae such as (1.9) completely precise. Briefly, the contour integral over $x$ arises geometrically as an integral over the Cartan subalgebra of $S U(2)$, regarded as the group of constant gauge transformations on $S^{3}$. The constant gauge transformations are the stabilizer of the trivial connection in the group $\mathcal{G}$ of all gauge transformations, and the presence of this stabilizer group plays an important role in the semi-classical analysis of the Wilson loop path integral. Moreover, all dependence on the $S U(2)$ representation $\boldsymbol{j}$ enters the integrand of (1.9) through the character $\mathrm{ch}_{\boldsymbol{j}}$. As a result, the character $\mathrm{ch}_{\boldsymbol{j}}$ can be naturally interpreted the avatar of the unknot Wilson loop operator itself when the path integral in (1.2) is reduced to the contour integral in (1.9).

As an aside, let me mention two interesting generalizations of the semi-classical formula for $Z(k ; \bigcirc, j)$ in (1.9). First, this formula extends directly to gauge groups $G$ other than $S U(2)$, in which case the unknot Wilson loop operator for any irreducible representation $R$ of $G$ reduces naturally to the corresponding character $\operatorname{ch}_{R}$. Moreover, the semi-classical formula in (1.9) generalizes in a surprisingly simple way to the arbitrary $(\mathbf{p}, \mathbf{q})$-torus $\operatorname{knot} K_{\mathbf{p}, \mathbf{q}}$ in $S^{3}$,

$$
\begin{aligned}
& Z\left(k ; K_{\mathbf{p}, \mathbf{q}}, \boldsymbol{j}\right)=\frac{1}{2 \pi i} \frac{1}{\sqrt{\mathbf{p q}}} \exp \left[-\frac{i \pi}{2(k+2)}\left(\frac{\mathbf{p}}{\mathbf{q}}+\frac{\mathbf{q}}{\mathbf{p}}+\mathbf{p q}\left(j^{2}-1\right)\right)\right] \times \\
& \times \int_{-\infty}^{+\infty} d x \operatorname{ch}_{\boldsymbol{j}}\left(\mathrm{e}^{\frac{i \pi}{4}} \frac{x}{2}\right) \sinh \left(\mathrm{e}^{\frac{i \pi}{4}} \frac{x}{2 \mathbf{p}}\right) \sinh \left(\mathrm{e}^{\frac{i \pi}{4}} \frac{x}{2 \mathbf{q}}\right) \exp \left[-\frac{(k+2)}{8 \pi}\left(\frac{x^{2}}{\mathbf{p q}}\right)\right] .
\end{aligned}
$$

Modulo a rather subtle overall phase, the integrand in (1.11) merely acquires denominators proportional to $\mathbf{p}$ and $\mathbf{q}$. From the semi-classical perspective, these denominators are associated to the $U(1)$ stabilizers of the exceptional Seifert fibers in $S^{3}$. I refer the interested reader to $\S 7.2$ in [5] for a detailed discussion of (1.11).

\section{Non-Abelian Localization}

My goal here is to explain a second, more conceptual way to understand the exactness of the stationary-phase approximation to the Seifert loop path integral. In this approach, we apply non-abelian localization, as introduced by Witten in [26], to study Chern-Simons theory on a Seifert manifold.

Very briefly, non-abelian localization provides a cohomological interpretation for a special class of symplectic integrals which are intimately related to symmetries. These integrals take the canonical form

$$
Z(\epsilon)=\int_{X} \exp \left[\Omega-\frac{1}{2 \epsilon}(\mu, \mu)\right]
$$

Here $X$ is an arbitrary symplectic manifold with symplectic form $\Omega$. We assume that a Lie group $H$ acts on $X$ in a Hamiltonian fashion with moment map $\mu: X \rightarrow \mathfrak{h}^{*}$, where $\mathfrak{h}^{*}$ is the dual of the Lie algebra $\mathfrak{h}$ of $H$. We also introduce an invariant 
quadratic form $(\cdot, \cdot)$ on $\mathfrak{h}$ and dually on $\mathfrak{h}^{*}$ to define the function $S=\frac{1}{2}(\mu, \mu)$ appearing in the integrand of $Z(\epsilon)$. Finally, $\epsilon \in \mathbb{R}$ is a coupling parameter 4

The symplectic integral in (1.12) has a number of important properties, which for sake of brevity I merely state. See [21, 26 for proofs of the following statements. First, the integrand of (1.12) admits an interpretation in terms of the $H$-equivariant cohomology ring of $X$. Using this interpretation, one can then show that the symplectic integral itself localizes onto the critical points of the invariant function $S=\frac{1}{2}(\mu, \mu)$ on $X$. Moreover, a non-abelian localization formula, roughly analogous to the Duistermaat-Heckman formula $\mathbf{9}$, exists to describe the contributions from the critical locus of $S$. In a smooth situation, these contributions are given by the integrals of certain de Rham cohomology classes over the critical loci. See $\S 6$ of 5] for a precise statement of the non-abelian localization formula applicable to Chern-Simons theory.

\section{Example: Two-Dimensional Yang-Mills Theory}

Given the special form of $Z(\epsilon)$, one should not be surprised that this integral has special properties. But why consider such an integral in the first place? One answer, following Witten [26, is that the path integral of two-dimensional YangMills theory assumes precisely the canonical symplectic form in (1.12).

To explain the latter observation, I will simply exhibit the counterparts of $X, \Omega, H$, and $\mu$ relevant to describe Yang-Mills theory on a Riemann surface $\Sigma$. The Yang-Mills path integral is formally an integral over the affine space $\mathcal{A}$ of connections on a fixed principal $G$-bundle $P$ over $\Sigma$, so clearly we must set

$$
X=\mathcal{A} \text {. }
$$

The affine space $\mathcal{A}$ also possesses a natural symplectic form $\Omega$ given by the intersection pairing on $\Sigma$,

$$
\Omega=-\int_{\Sigma} \operatorname{Tr}(\delta A \wedge \delta A) .
$$

Here $\delta$ denotes the exterior derivative acting on $\mathcal{A}$. Since $A$ serves as a coordinate on $\mathcal{A}, \delta A$ is a one-form on $\mathcal{A}$, and $\Omega$ is a two-form on $\mathcal{A}$ which is manifestly nondegenerate and closed. Of course on $\Sigma$ itself, $\delta A$ transforms as a section of the bundle $\Omega_{\Sigma}^{1} \otimes \operatorname{ad}(P)$ of adjoint-valued one-forms.

The obvious group which acts on $\mathcal{A}$ is the group $\mathcal{G}$ of gauge transformations. As shown long ago by Atiyah and Bott 2, the action of $\mathcal{G}$ on $\mathcal{A}$ is Hamiltonian with moment map given by the curvature $F_{A}=d A+A \wedge A$. That is, since elements in the Lie algebra of $\mathcal{G}$ appear on $\Sigma$ as sections of the adjoint bundle ad $(P)$, the curvature $F_{A}$, as a section of $\Omega_{\Sigma}^{2} \otimes \operatorname{ad}(P)$, can naturally be considered as a function on $\mathcal{A}$ taking values in the dual of the Lie algebra of $\mathcal{G}$. Thus,

$$
H=\mathcal{G}, \quad \mu=F_{A} .
$$

Finally, the Lie algebra of $\mathcal{G}$ admits an invariant form given by

$$
(\phi, \phi)=-\int_{\Sigma} \operatorname{Tr}(\phi \wedge \star \phi) .
$$

\footnotetext{
${ }^{4}$ To make sense of the measure on $X$ in 1.12 , we expand the exponential $\exp (\Omega)$ in its Taylor series and pick out the term $\frac{1}{n !} \Omega^{n}$ of proper degree to integrate over $X$. Hence $\exp (\Omega)$ conveniently describes the symplectic measure on $X$.
} 
Here $\phi$ is an element of the Lie algebra of $\mathcal{G}$, transforming on $\Sigma$ as a section of $\operatorname{ad}(P)$. With the quadratic form in (1.16), the invariant function $S=\frac{1}{2}(\mu, \mu)$ appearing in the canonical symplectic integral over $\mathcal{A}$ immediately becomes the Yang-Mills action,

$$
S=\frac{1}{2}(\mu, \mu)=-\frac{1}{2} \int_{\Sigma} \operatorname{Tr}\left(F_{A} \wedge \star F_{A}\right) .
$$

The metric on $\operatorname{Lie}(\mathcal{G})$ in (1.16) is defined using a duality operator $\star$ on $\Sigma$. For two-dimensional Yang-Mills theory, $\star$ relates zero-forms to two-forms, and to obtain such an operator, we only require a symplectic structure, as opposed to a metric, on $\Sigma$. Given a symplectic form $\omega$ on $\Sigma$, we define $\star$ by the condition $\star 1=\omega$. The symplectic form $\omega$ is invariant under all area-preserving diffeomorphisms of $\Sigma$, and this large group acts as a symmetry of two-dimensional Yang-Mills theory. As a result, two-dimensional Yang-Mills theory is essentially a topological gauge theory.

In the remainder of this article, I want to explain how to recast the Seifert loop path integral (1.2) as a symplectic integral of the canonical form (1.12). Once this step is accomplished, the general arguments in 26 imply that the Seifert loop path integral localizes onto critical points of the classical action $S=\frac{1}{2}(\mu, \mu)$. Furthermore, using the non-abelian localization formula, one can perform exact computations of the Seifert loop path integral and thus obtain a cohomological description for the Seifert loop operator itself. Specifically, as demonstrated in $§ 7.3$ of [5], the Seifert loop operator reduces naturally to the Chern character of an associated universal bundle over the moduli space of flat connections on $M$.

Two key ideas are required to obtain a symplectic description of the Seifert loop path integral. The first idea, which appears in $\S 3$ of $[4$, pertains to the basic Chern-Simons path integral in (1.5) and really has nothing to do with the Wilson loop operator. In contrast, the second idea concerns the Wilson loop operator itself and really has nothing to do with Chern-Simons theory. Nonetheless, both of these ideas fit together in a very elegant way.

\section{The Symplectic Geometry of Chern-Simons Theory}

The path integral which describes the partition function of two-dimensional Yang-Mills theory automatically assumes the canonical symplectic form in (1.12). As a special case of our general study of $Z(k ; C, R)$, I now want to review how the path integral (1.5) which describes the partition function $Z(k)$ of Chern-Simons theory on a Seifert manifold $M$ can also be cast as such a symplectic integral.

In order to obtain a symplectic interpretation of the two-dimensional YangMills path integral, we found it necessary to introduce a symplectic structure on $\Sigma$. To obtain a corresponding symplectic interpretation for the Chern-Simons path integral, we must introduce the analogous geometric structure on the three-manifold $M$ - namely, a contact structure.

Globally, a contact structure on $M$ is described by an ordinary one-form $\kappa$, a section of $\Omega_{M}^{1}$, which at each point of $M$ satisfies the contact condition

$$
\kappa \wedge d \kappa \neq 0 .
$$

By a classic theorem of Martinet [18, any compact, orientable 5 three-manifold admits a contact structure, so we do not necessarily assume $M$ to be Seifert at

\footnotetext{
${ }^{5}$ Any three-manifold admitting a contact structure must be orientable, since the nowhere vanishing three-form $\kappa \wedge d \kappa$ defines an orientation.
} 
this stage. However, if $M$ is a Seifert manifold, then we certainly want the contact form $\kappa$ to respect the $U(1)$ action on $M$. Such a contact form can be exhibited as follows.

We recall that the Seifert manifold $M$ is the total space of an $S^{1}$-bundle of degree $n$ over $\Sigma$,

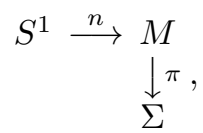

or an orbifold version thereof. For simplicity, I will phrase the following construction of $\kappa$ in the language of smooth manifolds, but the orbifold generalization is immediate.

Regarding $M$ as the total space of a principal $U(1)$-bundle, we take $\kappa$ to be a $U(1)$-connection on this bundle which satisfies

$$
d \kappa=n \pi^{*}(\omega) .
$$

Here $\omega$ is any unit-area symplectic form on $\Sigma$, and we recall that a $U(1)$-connection on $\Sigma$ appears upstairs on $M$ as an ordinary one-form. Because $d \kappa$ represents the Euler class of the $S^{1}$-bundle over $\Sigma$, the degree $n$ necessarily appears in (2.3). As an abelian connection, $\kappa$ is automatically invariant under the $U(1)$-action on $M$. Also, since the pullback of $\kappa$ to each $S^{1}$ fiber is non-vanishing, the contact condition in (2.1) is satisfied so long as $n \neq 0$ and the bundle is non-trivial, as we assume.

Chern-Simons theory is often considered to be an intrinsically three-dimensional gauge theory. However, one of the more interesting results in $\mathbf{4}, \mathbf{5}$ is to show that Chern-Simons theory is not quite a three-dimensional gauge theory, since one of the three components of $A$ can be completely decoupled from all topological observables.

In order to decouple one component of $A$ from the Chern-Simons path integral, we introduce a new, infinite-dimensional "shift" symmetry $\mathcal{S}$ which acts on $A$ as

$$
\delta A=\sigma \kappa .
$$

Here $\sigma$ is an arbitrary adjoint-valued scalar, a section of $\Omega_{M}^{1} \otimes \mathfrak{g}$, that parametrizes the action of $\mathcal{S}$ on $\mathcal{A}$.

Of course, the Chern-Simons action CS( $\cdot)$ does not respect the shift of $A$ in (2.4), so we must play a little path integral trick, of the sort familiar from path integral derivations of $T$-duality or abelian $S$-duality. See $\S 8$ in [27 for a nice review of the path integral derivations of these dualities.

We first introduce a new field $\Phi$ which transforms like $\sigma$ as an adjoint-valued scalar, a section of $\Omega_{M}^{1} \otimes \mathfrak{g}$, and which is completely gauge-trivial under $\mathcal{S}$. Thus $\mathcal{S}$ acts on $\Phi$ as

$$
\delta \Phi=\sigma .
$$

Next, we consider a new, shift-invariant action $S(A, \Phi)$ incorporating both $A$ and $\Phi$ such that, if $\Phi$ is set identically to zero via (2.5), then $S(A, \Phi)$ reduces to the Chern-Simons action for $A$. This condition fixes $S(A, \Phi)$ to be

$$
\begin{aligned}
S(A, \Phi) & =\operatorname{CS}(A-\kappa \Phi), \\
& =\operatorname{CS}(A)-\int_{M}\left[2 \kappa \wedge \operatorname{Tr}\left(\Phi F_{A}\right)-\kappa \wedge d \kappa \operatorname{Tr}\left(\Phi^{2}\right)\right] .
\end{aligned}
$$


Finally, using (2.6) we introduce an a priori new path integra 6 over both $A$ and $\Phi$,

$$
\widetilde{Z}(k)=\int \mathcal{D} A \mathcal{D} \Phi \exp \left[i \frac{k}{4 \pi} S(A, \Phi)\right] .
$$

On one hand, if we set $\Phi \equiv 0$ using the shift-symmetry in (2.5), $\widetilde{Z}(k)$ immediately reduces 7 to the usual Chern-Simons partition function $Z(k)$. Hence,

$$
\widetilde{Z}(k)=Z(k) .
$$

On the other hand, due to the elementary fact that $\kappa \wedge \kappa=0$, the field $\Phi$ appears only quadratically in $S(A, \Phi)$. So we can simply perform the Gaussian integral over $\Phi$ in (2.7) to obtain a new path integral description of the Chern-Simons partition function,

$$
Z(k)=\int \mathcal{D} A \exp \left[i \frac{k}{4 \pi} S(A)\right]
$$

where

$$
S(A)=\operatorname{CS}(A)-\int_{M} \frac{1}{\kappa \wedge d \kappa} \operatorname{Tr}\left[\left(\kappa \wedge F_{A}\right)^{2}\right] .
$$

In performing the Gaussian integral over $\Phi$, we use the contact condition on $\kappa$ in (2.1), since this condition ensures that quadratic term for $\Phi$ in (2.6) is everywhere non-degenerate on $M$. In particular, the inverse " $1 / \kappa \wedge d \kappa$ " appearing in (2.10) is defined as follows. Because $\kappa \wedge d \kappa$ is everywhere non-vanishing, we can always write $\kappa \wedge F_{A}=\varphi \kappa \wedge d \kappa$ for some section $\varphi$ of $\Omega_{M}^{0} \otimes \mathfrak{g}$. Thus, we set $\kappa \wedge F_{A} / \kappa \wedge d \kappa=\varphi$, and the second term in $S(A)$ becomes $\int_{M} \kappa \wedge \operatorname{Tr}\left(F_{A} \varphi\right)$.

By construction, $S(A)$ is invariant under the shift of $A$ in (2.4), as can be checked directly. Thus, we have obtained a new description of the Chern-Simons partition function for which one component of $A$ completely decouples from the path integral. Further, we have yet to use the condition that $M$ be a Seifert manifold, so the reformulation of $Z(k)$ via the shift-invariant action in (2.10) holds for any three-manifold $M$ endowed with a contact structure. See [14] for a detailed analysis of the shift-invariant reformulation of $Z(k)$ in the special case that the gauge group is $U(1)$.

\section{Symplectic Data}

If $M$ is a Seifert manifold, an additional miracle occurs, and the path integral in (2.9) becomes an integral of the canonical symplectic form to which non-abelian localization applies. For sake of time, let me merely summarize the symplectic data associated to (2.9).

First, the space over which we integrate in (2.9) and which must play the role of $X$ is the quotient of the affine space $\mathcal{A}$ by the group $\mathcal{S}$,

$$
X=\mathcal{A} / \mathcal{S} .
$$

In three dimensions, the affine space $\mathcal{A}$ is not symplectic. However, once we take the quotient by $\mathcal{S}$ in (2.11), the space $\mathcal{A} / \mathcal{S}$ carries a natural symplectic form $\Omega$

\footnotetext{
${ }^{6}$ I will not be very careful about the overall normalizations for the path integrals that appear here, but see $\S 3$ of [4] for a detailed accounting of formal normalization factors.

${ }^{7}$ We note that the Jacobian associated to the gauge-fixing condition $\Phi \equiv 0$ for $\mathcal{S}$ is trivial.
} 
given by

$$
\Omega=-\int_{M} \kappa \wedge \operatorname{Tr}(\delta A \wedge \delta A)
$$

Clearly $\Omega$ is both gauge-invariant and shift-invariant, and $\Omega$ descends to a nondegenerate symplectic form on $\mathcal{A} / \mathcal{S}$.

We must now find a Hamiltonian group acting on $\mathcal{A} / \mathcal{S}$ such that the shiftinvariant action $S(A)$ is the square of the corresponding moment map. As an initial guess, motivated by the example of two-dimensional Yang-Mills theory, one might consider the group $\mathcal{G}$ of gauge transformations. However, this guess cannot be correct. By construction, the square of the moment map for the action of $\mathcal{G}$ on $\mathcal{A} / \mathcal{S}$ would be invariant under $\mathcal{G}$. However, $S(A)$ is plainly not invariant under $\mathcal{G}$, since the Chern-Simons term appearing in (2.10) is not invariant under the large gauge transformations in $\mathcal{G}$ (while the remaining term in (2.10) is manifestly invariant under $\mathcal{G})$.

As a second guess, one might replace $\mathcal{G}$ with its identity component $\mathcal{G}_{0}$, which does preserve the shift-invariant action $S(A)$. However, one can show that $\mathcal{G}_{0}$ is obstructed from acting in a Hamiltonian fashion on $\mathcal{A} / \mathcal{S}$ by a non-trivial Lie algebra cocycle $c$,

$$
c(\phi, \psi)=-\int_{M} d \kappa \wedge \operatorname{Tr}(\phi d \psi) .
$$

Here $\phi$ and $\psi$ are elements of the Lie algebra of $\mathcal{G}_{0}$, transforming as sections of $\Omega_{M}^{0} \otimes \mathfrak{g}$ on $M$. Parenthetically, this cocycle is closely related to a cocycle that appears in the theory of loop groups $[\mathbf{2 2}$, which also provides useful background for the identification of $H$ below.

To remedy the situation, we consider the central extension $8 \widetilde{\mathcal{G}}_{0}$ of $\mathcal{G}_{0}$ determined by $c(\phi, \psi)$,

$$
U(1)_{\mathrm{Z}} \stackrel{c}{\longrightarrow} \widetilde{\mathcal{G}}_{0} \longrightarrow \mathcal{G}_{0} .
$$

Here we use the subscript ' $\mathrm{Z}$ ' to emphasize that $U(1)_{\mathrm{Z}}$ is central in $\widetilde{\mathcal{G}}_{0}$. The natural action of $\mathcal{G}_{0}$ on $\mathcal{A} / \mathcal{S}$ extends to an action by $\widetilde{\mathcal{G}_{0}}$, for which $U(1)_{\mathrm{Z}}$ acts trivially. By construction, the action of $\widetilde{\mathcal{G}}_{0}$ on $\mathcal{A} / \mathcal{S}$ is then Hamiltonian.

However, $\widetilde{\mathcal{G}}_{0}$ is still not the group which is to play the role of the Hamiltonian group $H$ ! In order to define the canonical symplectic integral in (1.12), the Lie algebra of $H$ must carry a non-degenerate, invariant quadratic form $(\cdot, \cdot)$. But the Lie algebra of $\widetilde{\mathcal{G}_{0}}$ does not admit such a form, essentially because we have no generator to pair with the generator of the central $U(1)_{\mathrm{Z}}$.

However, we have also yet to apply the Seifert condition on $M$. We do so now. To avoid confusion, let me denote the Seifert $U(1)$ acting on $M$ by $U(1)_{\mathrm{R}}$, to distinguish it from the central $U(1)_{\mathrm{z}}$. The action by $U(1)_{\mathrm{R}}$ on $M$ induces a corresponding action on both $\widetilde{\mathcal{G}}_{0}$ and $\mathcal{A} / \mathcal{S}$, and we finally take $H$ to be the semidirect product

$$
H=U(1)_{\mathrm{R}} \ltimes \widetilde{\mathcal{G}}_{0} .
$$

\footnotetext{
${ }^{8} \mathrm{~A}$ Lie algebra two-cocycle always determines a central extension of algebras. Provided that the cocycle is properly quantized, as is the cocycle in (2.13), the central extension of algebras lifts to a corresponding central extension of groups.
} 
The Lie algebra of $H$ does admit a non-degenerate, invariant quadratic form $(\cdot, \cdot)$, under which the generators of $U(1)_{\mathrm{R}}$ and $U(1)_{\mathrm{Z}}$ are paired. Furthermore, the action of $H$ on $\mathcal{A} / \mathcal{S}$ is Hamiltonian with moment map $\mu$ (for which a completely explicit though perhaps not so illuminating formula exists), and the corresponding invariant function $S=\frac{1}{2}(\mu, \mu)$ on $\mathcal{A} / \mathcal{S}$ is precisely $S(A)$. Given the amount of symmetry respected by both $\frac{1}{2}(\mu, \mu)$ and $S(A)$, the latter result could hardly have been otherwise.

The presentation here is a regrettably quick sketch of a fairly miraculous result, and I refer the reader to $\S 3$ of 4 ] for a complete discussion.

\section{Inclusion of Wilson Loop Operators}

I now want to explain how the prior statements concerning the Chern-Simons partition function can be generalized to allow for insertions of Wilson loop operators. (See $\S 4$ of $[\mathbf{5}$ for an expanded version of the material here.) As it happens, only one new idea is required.

We clearly need a new idea, because a naive attempt to reapply the previous path integral manipulations to the Wilson loop path integral in (1.2) runs immediately aground. To illustrate the difficulty with the direct approach, let us consider the obvious way to rewrite the Wilson loop path integral in a shift-invariant form,

$$
Z(k ; C, R)=\int \mathcal{D} A \mathcal{D} \Phi \mathcal{W}_{R}(C) \exp \left[i \frac{k}{4 \pi} \operatorname{CS}(A-\kappa \Phi)\right] .
$$

Here $\mathcal{W}_{R}(C)$ denotes the generalized Wilson loop operator defined not using $A$ but using the shift-invariant combination $A-\kappa \Phi$, so that

$$
\mathcal{W}_{R}(C)=\operatorname{Tr}_{R} P \exp \left[-\oint_{C}(A-\kappa \Phi)\right] .
$$

Exactly as for our discussion of (2.7), we can use the shift symmetry to fix $\Phi \equiv 0$, after which the path integral in (3.1) reduces trivially to the standard Wilson loop path integral in (1.2).

However, to learn something useful from (3.1) we must perform the path integral over $\Phi$, and as it stands, this integral is not easy to do. Because the generalized Wilson loop operator $\mathcal{W}_{R}(C)$ is expressed in (3.2) as a complicated, non-local functional of $\Phi$, the path integral over $\Phi$ in (3.1) is not a Gaussian integral that we can trivially evaluate as we did for (2.7).

A more fundamental perspective on our problem is the following. Let us return to the description of the ordinary Wilson loop operator $W_{R}(C)$ as the trace in the representation $R$ of the holonomy of $A$ around $C$,

$$
W_{R}(C)=\operatorname{Tr}_{R} P \exp \left(-\oint_{C} A\right) \text {. }
$$

As observed by Witten in one of the small gems of [24, this description of $W_{R}(C)$ should be regarded as intrinsically quantum mechanical, for the simple reason that $W_{R}(C)$ can be naturally interpreted in (3.3) as the partition function of an auxiliary quantum system attached to the curve $C$. Briefly, the representation $R$ is to be identified with the Hilbert space of this system, the holonomy of $A$ is to be identified with the time-evolution operator around $C$, and the trace over $R$ is the usual trace over the Hilbert space that defines the partition function in the Hamiltonian formalism. 
Because the notion of tracing over a Hilbert space is inherently quantum mechanical, any attempts to perform essentially classical path integral manipulations involving the expressions in (3.2) or (3.3) are misguided at best. Rather, if we hope to generalize the semi-classical path integral manipulations which we used to study the Chern-Simons partition function, we need to use an alternative, semi-classical description for the Wilson loop operator itself.

More precisely, we want to replace the quantum mechanical trace over $R$ in (3.3) by a path integral over an auxiliary bosonic field $U$ which is attached to the curve $C$ and coupled to the connection $A$ as a background field, so that schematically

$$
W_{R}(C)=\int \mathcal{D} U \exp \left[i \operatorname{cs}_{\alpha}\left(U ;\left.A\right|_{C}\right)\right]
$$

Here $\operatorname{cs}_{\alpha}\left(U ;\left.A\right|_{C}\right)$ is an action, depending upon the representation $R$ through its highest weight $\alpha$, which is a local, gauge-invariant, and indeed topological functional of the defect field $U$ and the restriction of $A$ to $C$. Not surprisingly, this semiclassical description (3.4) of $W_{R}(C)$ turns out to be the key ingredient required to reformulate the Wilson loop path integral in a shift-invariant fashion.

The idea of representing the Wilson loop operator by a path integral as in (3.4) is a very old and very general piece of gauge theory lore. In the context of four-dimensional Yang-Mills theory, this idea goes back (at least) to work of Balachandran, Borchardt, and Stern [3] in the 1970's. See also [8] and $\S 7.7$ in [27] for other appearances of the path integral in (3.4).

The basic idea behind the path integral description (3.4) of the Wilson loop operator is very simple. We interpret the closed curve $C$ as a periodic "time" for the field $U$, and we apply the Hamiltonian formalism to rewrite the path integral over $U$ axiomatically as the quantum mechanical trace of the corresponding time-evolution operator around $C$,

$$
W_{R}(C)=\operatorname{Tr}_{\mathscr{H}} P \exp \left(-i \oint_{C} \mathbf{H}\right) .
$$

Here $\mathscr{H}$ is the Hilbert space obtained by quantizing $U$, and $\mathbf{H}$ is the Hamiltonian which acts on $\mathscr{H}$ to generate infinitesimal translations along $C$.

Comparing the conventional description of the Wilson loop operator in (3.3) to the axiomatic expression in (3.5), we see that the two agree if we identify 9

$$
\begin{aligned}
& R \longleftrightarrow \mathscr{H}, \\
& P \exp \left(-\oint_{C} A\right) \\
& P \exp \left(-i \oint_{C} \mathbf{H}\right) .
\end{aligned}
$$

Hence to make the Wilson loop path integral in (3.4) precise, we need only exhibit a classical theory on $C$, for which the gauge group $G$ acts as a symmetry, such that upon quantization we obtain a Hilbert space $\mathscr{H}$ isomorphic to $R$ and for which the time-evolution operator around $C$ is given by the holonomy of $A$, acting as an element of $G$ on $R$.

\footnotetext{
${ }^{9}$ We follow the standard physical definition according to which $\mathbf{H}$ is a hermitian operator, accounting for the ' $-i$ ' in (3.5). We also recall that the gauge field $A$ is valued in the Lie algebra $\mathfrak{g}$, so $A$ is anti-hermitian and no ' $i$ ' appears in the holonomy.
} 


\section{A Semi-Classical Description of the Wilson Loop Operator}

Let me now tell you what classical theory to place on $C$ to realize the quantum identifications in (3.6).

Of the two identifications in (3.6), the more fundamental by far is the identification of the irreducible representation $R$ with a Hilbert space $\mathscr{H}$, obtained by quantizing some classical phase space upon which $G$ acts as a symmetry. So before we even consider what classical theory must live on $C$ to describe the Wilson loop operator, we can ask the simpler and more basic question - what classical phase space must we quantize to obtain $R$ as a Hilbert space?

As well-known, this question is beautifully answered by the Borel-Weil-Bott theorem [7. In order to recall this theorem, let me first fix a maximal torus $T \subset G$, for which $\mathfrak{t} \subset \mathfrak{g}$ is the associated Cartan subalgebra. Given the irreducible representation $R$ and some choice of positive roots for $G$, I also introduce the associated highest weight $\alpha$. Canonically, the weight $\alpha$ lies in the dual $\mathfrak{t}^{*}$ of $\mathfrak{t}$, but given the invariant form ' $\operatorname{Tr}$ ' on $\mathfrak{g}$, we are free to identify $\mathfrak{t}^{*} \cong \mathfrak{t}$ and hence to regard $\alpha$ as an element of $\mathfrak{t}$,

$$
\alpha \in \mathfrak{t}^{*} \cong \mathfrak{t} .
$$

Though mathematically unnatural, the convention in (3.7) proves to be convenient later.

The Borel-Weil-Bott theorem concerns the geometry of the orbit $\mathcal{O}_{\alpha} \subset \mathfrak{g}$ which passes through $\alpha$ under the adjoint action of $G$. Equivalently, the adjoint orbit $\mathcal{O}_{\alpha}$ can be realized as a quotient $G / G_{\alpha}$, where $G_{\alpha}$ is the stabilizer of $\alpha$ under the adjoint action of $G$. Explicitly, the identification between $G / G_{\alpha}$ and $\mathcal{O}_{\alpha}$ is given by the map

$$
g G_{\alpha} \longmapsto g \alpha g^{-1}, \quad g \in G .
$$

As will be essential in a moment, $\mathcal{O}_{\alpha}$ is a compact complex manifold which admits a natural Kähler structure invariant under $G$. For instance, if $G=S U(2)$ and $\alpha$ is any non-zerd 10 weight, then $\mathcal{O}_{\alpha}=S U(2) / U(1)$ can be identified as $\mathbb{C P}^{1}$ endowed with the round, Fubini-Study metric.

In a nutshell, the Borel-Weil-Bott theorem states that the irreducible representation $R$ can be realized geometrically as the space of holomorphic sections of a certain unitary line bundle $\mathfrak{L}(\alpha)$ over $\mathcal{O}_{\alpha}$. That is,

$$
R \cong H \frac{0}{\partial}\left(\mathcal{O}_{\alpha}, \mathfrak{L}(\alpha)\right)
$$

where the action of $G$ on the sections of $\mathfrak{L}(\alpha)$ is induced from its action on $\mathcal{O}_{\alpha}$.

As a unitary line bundle over a Kähler manifold, $\mathfrak{L}(\alpha)$ carries a natural unitary connection $\Theta_{\alpha}$ which is also invariant under $G$. The connection $\Theta_{\alpha}$ enters the path integral description of the Wilson loop operator, so let me exhibit it explicitly. When pulled back to $G$, the line bundle $\mathfrak{L}(\alpha)$ trivializes, and the connection $\Theta_{\alpha}$ appears as the following left-invariant one-form on $G$,

$$
\Theta_{\alpha}=\operatorname{Tr}\left(\alpha \cdot g^{-1} d g\right) \text {. }
$$

I have introduced the connection $\Theta_{\alpha}$ because its curvature $\nu_{\alpha}=d \Theta_{\alpha}$ is precisely the Kähler form on $\mathcal{O}_{\alpha}$. As a result, $\mathfrak{L}(\alpha)$ is a prequantum line bundle over $\mathcal{O}_{\alpha}$, and the Borel-Weil-Bott isomorphism in (3.9) identifies $R$ as the Hilbert space obtained by Kähler quantization of $\mathcal{O}_{\alpha}$.

\footnotetext{
${ }^{10}$ Of course, if $\alpha=0$, then $\mathcal{O}_{\alpha}$ is merely a point.
} 
Perhaps more physically, the Borel-Weil-Bott theorem can be interpreted as identifying the space of groundstates for a charged particle moving on $\mathcal{O}_{\alpha}$ in the presence of a background magnetic field given by $\nu_{\alpha}$. Briefly, because of the non-zero magnetic field, the wavefunctions which describe this particle transform on $\mathcal{O}_{\alpha}$ not as functions but as sections of the line bundle $\mathfrak{L}(\alpha)$. As standard, the Hamiltonian which describes free propagation on $\mathcal{O}_{\alpha}$ is proportional to the Laplacian $\triangle$ acting on sections of $\mathfrak{L}(\alpha)$, and by Hodge theory, the kernel of $\triangle$ can be identified as the space of holomorphic sections of $\mathfrak{L}(\alpha)$. Hence the role of (3.9) is to realize the representation $R$ in terms of groundstates on $\mathcal{O}_{\alpha}$.

Given the previous quantum mechanical interpretation for $R$, the corresponding path integral description (3.4) for the Wilson loop operator follows immediately. Ignoring the coupling to $A$ for a moment, if we simply wish to describe the lowenergy effective dynamics of an electron moving on $\mathcal{O}_{\alpha}$ in the background magnetic field $\nu_{\alpha}$, we consider a one-dimensional sigma model of maps

$$
U: C \longrightarrow \mathcal{O}_{\alpha},
$$

with sigma model action

$$
\operatorname{cs}_{\alpha}(U)=\oint_{C} U^{*}\left(\Theta_{\alpha}\right)=\oint_{C} \operatorname{Tr}\left(\alpha \cdot g^{-1} d g\right) .
$$

Here $U^{*}\left(\Theta_{\alpha}\right)$ denotes the pullback of $\Theta_{\alpha}$ to a connection over $C$. If $U$ is lifted as a map to $\mathcal{O}_{\alpha}=G / G_{\alpha}$ by a corresponding map

$$
g: C \longrightarrow G,
$$

then the pullback of $\Theta_{\alpha}$ appears explicitly as in (3.12). As a word of warning, I will freely switch between writing formulae in terms of $U$ or $g$ as convenient.

From a physical perspective, the first-order action $\mathrm{cs}_{\alpha}$ simply describes the minimal coupling of the charged particle on $\mathcal{O}_{\alpha}$ to the background magnetic field specified by $\Theta_{\alpha}$, and we have omitted the second-order kinetic terms for $U$ as being irrelevant at low energies. From a more geometric perspective, $\mathrm{cs}_{\alpha}$ is a onedimensional Chern-Simons action for the abelian connection $U^{*}\left(\Theta_{\alpha}\right)$ over $C$. As such, the quantization of the parameter $\alpha \in \mathfrak{t}$ as a weight of $G$ follows just as for the quantization of the Chern-Simons level $k$. More physically, the quantization of $\alpha$ follows from the quantization of magnetic flux on a compact space.

This sigma model on $C$ clearly respects the action of $G$ on $\mathcal{O}_{\alpha}$ as a global symmetry. To couple the sigma model to the restriction of the bulk gauge field $A$, we simply promote the global action of $G$ on $\mathcal{O}_{\alpha}$ to a gauge symmetry. That is, we consider the covariant version of (3.12),

$$
\begin{aligned}
\operatorname{cs}_{\alpha}\left(U ;\left.A\right|_{C}\right) & =\oint_{C} U^{*}\left(\Theta_{\alpha}(A)\right)=\oint_{C} \operatorname{Tr}\left(\alpha \cdot g^{-1} d_{A} g\right), \\
d_{A} g & =d g+\left.A\right|_{C} \cdot g .
\end{aligned}
$$

In the second line, we indicate the action of the covariant derivative $d_{A}$ on $g$. The action by $d_{A}$ on $g$ descends to a corresponding covariant action by $d_{A}$ on $U$ as well.

I now claim that the quantization of the gauged sigma model on $C$ with action $\operatorname{cs}_{\alpha}\left(U ;\left.A\right|_{C}\right)$ leads to the identifications in (3.6) required to describe the Wilson loop operator. First, the classical equation of motion for $U$ simply asserts that $U$ is covariantly constant,

$$
d_{A} U=0 .
$$


As a result, the classical phase space for $U$ can be identified with the orbit $\mathcal{O}_{\alpha}$, and by the Borel-Weil-Bott isomorphism in (3.9), the corresponding Hilbert space $\mathscr{H}$ for $U$ is identified as the representation $R$. Similarly, since the classical timeevolution for $U$ is given by parallel transport, the quantum time-evolution operator around $C$ is immediately given by the holonomy of $A$, acting as an element of $G$ on $R$.

\section{The Shift-Invariant Wilson Loop in Chern-Simons Theory}

Because $A$ only enters as a background field in (3.14), the path integral description (3.4) of $W_{R}(C)$ is completely general and applies to any gauge theory in any dimension. Nonetheless, this description of the Wilson loop operator is precisely what we need to obtain a shift-invariant formulation of the Wilson loop path integral in Chern-Simons theory.

Let us first apply (3.4) to rewrite the Wilson loop path integral in (1.2) as a path integral over both $A$ and $U$,

$$
Z(k ; C, R)=\frac{1}{\operatorname{Vol}(\mathcal{G})} \int_{\mathcal{A} \times L \mathcal{O}_{\alpha}} \mathcal{D} A \mathcal{D} U \exp \left[i \frac{k}{4 \pi} \operatorname{CS}(A)+i \operatorname{cs}_{\alpha}\left(U ;\left.A\right|_{C}\right)\right] .
$$

Here we introduce the free loopspace $L \mathcal{O}_{\alpha}$ of $\mathcal{O}_{\alpha}$ to parametrize configurations of $U$.

Once we introduce the defect field $U$ coupling to $A$ in (3.16), the classical equation of motion for $A$ becomes

$$
\mathcal{F}_{A} \stackrel{\text { def }}{=} F_{A}+\frac{2 \pi}{k} U \cdot \delta_{C}=0 .
$$

Here $\delta_{C}$ is a two-form with delta-function support on $C$ which represents the Poincaré dual of the curve. Using $\delta_{C}$, we rewrite $\operatorname{cs}_{\alpha}\left(U ;\left.A\right|_{C}\right)$ as a bulk integral over $M$,

$$
\operatorname{cs}_{\alpha}\left(U ;\left.A\right|_{C}\right)=\oint_{C} \operatorname{Tr}\left(\alpha \cdot g^{-1} d_{A} g\right)=\int_{M} \delta_{C} \wedge \operatorname{Tr}\left(\alpha \cdot g^{-1} d_{A} g\right)
$$

from which (3.17) follows.

As we see from (3.17), in the presence of the operator $W_{R}(C)$, classical configurations for $A$ are given by connections which are flat on the knot complement

$$
M^{o}=M-C,
$$

and otherwise have delta-function curvature along $C$. The singularity in $A$ along $C$ manifests itself on $M^{o}$ as a non-trivial monodromy of the connection around a transverse circle linking $C$. Though I will not have time to say more, the moduli space of such flat connections with monodromies is the space onto which the Seifert loop path integral localizes. This space is directly related to the moduli space of representations of the knot group of $C$ in $G$ and, in suitable circumstances, fibers over the moduli space of (non-singular) flat connections on $M$.

At the cost of introducing defect degrees-of-freedom along $C$, we have managed to describe $W_{R}(C)$ in terms of a completely local - and indeed linear - functional of $A$. Consequently, the same path integral trick that we used to decouple one component of $A$ from the Chern-Simons partition function applies immediately to (3.16). We simply replace $A$ in (3.16) by the shift-invariant 11 combination $A-\kappa \Phi$,

\footnotetext{
${ }^{11}$ The shift-symmetry $\mathcal{S}$ acts trivially on $U$.
} 
and we then perform the Gaussian integral over $\Phi$. In the process, the only new ingredient is that we obtain an extra term linear in $\Phi$ from the coupling of $A$ to $U$.

Without discussing any more details, let me present the resulting shift-invariant formulation for the Wilson loop path integral,

$$
Z(k ; C, R)=\frac{1}{\operatorname{Vol}(\mathcal{G})} \int_{\mathcal{A} / \mathcal{S} \times L \mathcal{O}_{\alpha}} \mathcal{D} A \mathcal{D} U \exp \left[i \frac{k}{4 \pi} S(A, U)\right],
$$

where

$$
S(A, U)=\operatorname{CS}(A)+\frac{4 \pi}{k} \operatorname{cs}_{\alpha}\left(U ;\left.A\right|_{C}\right)-\int_{M} \frac{1}{\kappa \wedge d \kappa} \operatorname{Tr}\left[\left(\kappa \wedge \mathcal{F}_{A}\right)^{2}\right] .
$$

The shift-invariant action $S(A, U)$ appears much as the shift-invariant action (2.10) for $A$ alone, with the replacement therein of $F_{A}$ by the generalized curvature $\mathcal{F}_{A}$. Thus for an arbitrary Wilson loop (or link) operator in Chern-Simons theory, the path integral can be rewritten such that one component of $A$ completely decouples.

Moreover, if $M$ is a Seifert manifold and $C$ is a Seifert fiber of $M$, the shiftinvariant Seifert loop path integral is again an integral of the canonical symplectic form in (1.12). The relevant symplectic space $X$ is just the product

$$
X=\mathcal{A} / \mathcal{S} \times L \mathcal{O}_{\alpha},
$$

where the loopspace $L \mathcal{O}_{\alpha}$ carries a natural symplectic (and indeed Kähler) form inherited from the Kähler form $\nu_{\alpha}$ on $\mathcal{O}_{\alpha}$. The Hamiltonian group $H$ which acts on $X$ is the same group that appears in (2.15). In fact, the loopspace $L \mathcal{O}_{\alpha}$ can be interpreted formally as an infinite-dimensional coadjoint orbit of $H$. Finally, the square of the moment map for the diagonal action of $H$ on $X$ is precisely the shift-invariant action $S(A, U)$ appearing in (3.21).

For a last bit of furious hand-waving, let me remark that the description of the Seifert loop operator as a character follows quite naturally from the appearance of the loopspace $L \mathcal{O}_{\alpha}$ in (3.20). In essence, non-abelian localization on $L \mathcal{O}_{\alpha}$ is related to index theory on $\mathcal{O}_{\alpha}$, and index theory on $\mathcal{O}_{\alpha}$ provides a classic derivation [1] of the famous Weyl character formula. See $\S 7.2$ in [5] for a complete discussion.

\section{Acknowledgements}

I take pleasure in thanking the organizers and participants of the Bonn workshop "Chern-Simons Gauge Theory: 20 Years After," where this article was presented. I especially thank Edward Witten, both for our prior collaboration on the subject and for posing the question which sparked this work.

\section{References}

[1] M. Atiyah and R. Bott, "A Lefschetz Fixed Point Formula for Elliptic Complexes, II," Ann. of Math. 88 (1968) 451-491.

[2] M. Atiyah and R. Bott, "Yang-Mills Equations Over Riemann Surfaces," Phil. Trans. R. Soc. Lond. A308 (1982) 523-615.

[3] A. Balachandran, S. Borchardt, and A. Stern, "Lagrangian And Hamiltonian Descriptions of Yang-Mills Particles," Phys. Rev. D 17 (1978) 3247-3256.

[4] C. Beasley and E. Witten, "Non-Abelian Localization for Chern-Simons Theory," J. Diff. Geom. 70 (2005) 183-323, hep-th/0503126

[5] C. Beasley, "Localization for Wilson Loops in Chern-Simons Theory," arXiv:0911.2687 [hep-th].

[6] M. Blau and G. Thompson, "Chern-Simons Theory on $S^{1}$-Bundles: Abelianisation and qDeformed Yang-Mills Theory," JHEP 0605 (2006) 003, arXiv:hep-th/0601068 
[7] R. Bott, "Homogeneous Vector Bundles," Ann. of Math. 66 (1957) 203-248.

[8] D. Diakonov and V. Y. Petrov, "A Formula for the Wilson Loop," Phys. Lett. B 224 (1989) 131-135.

[9] J. J. Duistermaat and G. J. Heckman, "On the Variation in the Cohomology of the Symplectic Form of the Reduced Phase Space," Invent. Math. 69 (1982) 259-268; Addendum, Invent. Math. 72 (1983) 153-158.

[10] D. Freed and R. Gompf, "Computer Calculation of Witten's 3-Manifold Invariant," Commun. Math. Phys. 141 (1991) 79-117.

[11] S. Garoufalidis, Relations Among 3-Manifold Invariants, Ph.D. thesis, University of Chicago, 1992.

[12] S. Hansen and T. Takata, "Reshetikhin-Turaev Invariants of Seifert Three-Manifolds for Classical Simple Lie Algebras and Their Asymptotic Expansions," J. Knot Theory Ramifications 13 (2004) 617-668, math.GT/0209403

[13] L. Jeffrey, "Chern-Simons-Witten Invariants of Lens Spaces and Torus Bundles, and the Semiclassical Approximation," Commun. Math. Phys. 147 (1992) 563-604.

[14] L. Jeffrey and B. McLellan, "Eta-Invariants and Anomalies in U(1) Chern-Simons Theory," arXiv:1004.2913 [math.SG].

[15] A. Kapustin, B. Willett, and I. Yaakov, "Exact Results for Wilson Loops in Superconformal Chern-Simons Theories with Matter," arXiv:0909.4559 [hep-th].

[16] R. Lawrence and L. Rozansky, "Witten-Reshetikhin-Turaev Invariants of Seifert Manifolds," Commun. Math. Phys. 205 (1999) 287-314.

[17] M. Mariño, "Chern-Simons Theory, Matrix Integrals, and Perturbative Three-Manifold Invariants," Commun. Math. Phys. 253 (2004) 25-49, hep-th/0207096.

[18] J. Martinet, "Formes de contact sur les varietétés de dimension 3," Springer Lecture Notes in Math 209 (1971) 142-163.

[19] L. Moser, "Elementary Surgery Along a Torus Knot," Pacific J. Math 38 (1971) 737-745.

[20] "Problems on Invariants of Knots and 3-Manifolds," Ed. by T. Ohtsuki with an introduction by J. Roberts, in Invariants of Knots and 3-Manifolds (Kyoto, 2001), pp. 377-572, Geom. Topol. Monogr. 4, Geom. Topol. Publ., Coventry, 2002, math.GT/0406190

[21] P.-E. Paradan, "The Moment Map and Equivariant Cohomology with Generalized Coefficients," Topology 39 (2000) 401-444.

[22] A. Pressley and G. Segal, Loop Groups, Clarendon Press, Oxford, 1986.

[23] N. Reshetikhin and V. Turaev, "Invariants of 3-Manifolds via Link Polynomials and Quantum Groups," Invent. Math. 103 (1991) 547-597.

[24] E. Witten, "Quantum Field Theory and the Jones Polynomial," Commun. Math. Phys. 121 (1989) 351-399.

[25] E. Witten, "Gauge Theories and Integrable Lattice Models," Nucl. Phys. B 322 (1989) 629697.

[26] E. Witten, "Two-dimensional Gauge Theories Revisited," J. Geom. Phys. 9 (1992) 303-368, hep-th/9204083

[27] E.Witten, "Dynamics of Quantum Field Theory," in Quantum Fields and Strings: A Course for Mathematicians, Vol. 2, Ed. by P. Deligne et al., American Mathematical Society, Providence, Rhode Island, 1999.

Simons Center for Geometry and Physics, Stony Brook University, Stony Brook, NEW YoRK 11794-3636

E-mail address: cbeasley@insti.physics.sunysb.edu 